\title{
Advances in the role of autophagy in the development of retinoblastoma (Review)
}

\author{
TENG WAN ${ }^{1,2^{*}}$, MINGYUAN FU $^{2 *}$, ZHUAN WU $^{3 *}, \mathrm{XUE} \mathrm{GAO}^{1,4}$ and SHOUHONG ZHOU ${ }^{1,4}$ \\ ${ }^{1}$ Guangxi Key Laboratory of Brain and Cognitive Neuroscience, Guilin Medical University, Guilin, Guangxi 541199; \\ Departments of ${ }^{2}$ Physiology and ${ }^{3}$ Biochemistry, Hengyang Medical College, University of South China, Hengyang, \\ Hunan 421001; ${ }^{4}$ Department of Physiology, Basic Medical College; Guilin, Guangxi 541199, P.R. China
}

Received February 15, 2021; Accepted June 4, 2021

DOI: $10.3892 / \mathrm{ol} .2021 .12893$

\begin{abstract}
Autophagy is a feedback regulatory mechanism of cells to external stress, which helps cells to adapt to changes in physiological conditions and environmental stress. Autophagy possesses a variety of target genes that control a wide range of signaling pathways. Maintenance of an appropriate level of autophagy is essential for the growth, metastasis and characteristics of tumors. Retinoblastoma (RB) is the most common primary intraocular malignant tumor found in the eyes of children following exposure to extreme environmental factors, such as mitochondrial defects, oxidative stress and excessive autophagy; this leads to the development of DNA damage and progressive loss of the function of the eye, which results in the occurrence of RB. Recent studies have documented the involvement of autophagy in the transformation, occurrence and metastasis of RB. High or low levels of autophagy exert notably promotive or repressive effects on the development, invasion, drug resistance and survival of $\mathrm{RB}$, respectively. The present review reports the research progress on the association between autophagy and RB.
\end{abstract}

\section{Contents}

1. Introduction

2. Concept, classification, biological characteristics and regulation of autophagy

3. Autophagy and tumors

4. Association between autophagy and RB

5. Review and summary

Correspondence to: Dr Shouhong Zhou or Mrs. Xue Gao, Guangxi Key Laboratory of Brain and Cognitive Neuroscience, Guilin Medical University, 1 Zhiyuan Road, Guilin, Guangxi 541199, P.R. China

E-mail: 112019023@glmc.edu.cn; 723285991@qq.com

E-mail: 1336558223@qq.com

*Contributed equally

Key words: autophagy, tumor, retinoblastoma

\section{Introduction}

Retinoblastoma (RB) is the most common primary intraocular malignant tumor in children, with a constant incidence worldwide of 1:15,000-1:20,000 live births (1). At present, the treatment of RB mainly includes surgical enucleation, radiotherapy and chemotherapy (1). Due to its limited sensitivity to treatment, the prognosis of RB is often poor (1). RB is a disease caused by gene mutations. It has been demonstrated that the mutation of the RB1 gene located on $13 q 14$ plays a vital role in the development of RB. The RB1 gene participates in tumor inhibition, maintenance of gene stability and epigenetic inheritance. Therefore, unlimited proliferation of cells occurs when the RB1 gene is mutated (2).

Regarded as a catabolic process, autophagy is a response to cell nutrient deficiency, environmental stress and energy shortage, and plays an important role in clearing damaged organelles and aggregation of denatured proteins, as well as in recycling amino acids in the starvation state (3). Through various mechanisms, including degradation of damaged intracellular organelles and protection of DNA stability, autophagy maintains the normal function of cells and ensures gene stability to inhibit the occurrence of tumors $(4,5)$. However, in tumor cells, such as in RB, autophagy is the main resistance mechanism towards adverse factors, including an external anoxic environment or drug therapy, leading to the high invasion ability of tumors, an anti-anoxic environment and multidrug resistance $(6,7)$.

The present review describes the current research progress on the association between autophagy and $\mathrm{RB}$. The role and regulatory mechanism of autophagy in the occurrence, progression and treatment of $\mathrm{RB}$ are reported, and new strategies and targets for the treatment of RB are provided.

\section{Concept, classification, biological characteristics and regulation of autophagy}

Autophagy is associated with a variety of pathological and physiological processes. Generally, according to different mechanisms of action, autophagy is divided into three types, namely large autophagy, small autophagy and chaperonmediated autophagy $(8,9)$. Large autophagy is what the present review calls autophagy. Under particular conditions, such as 
lack of nutrients or energy, or oxygen deficiency, autophagy is induced to degrade cytoplasmic substances into metabolites in cells, thereby maintaining the normal survival of cells (10). In a previous study, the autophagy level was significantly decreased in mouse acinar cells lacking autophagy-related gene 7 (ATG7) (11), and further experimentation conclusively showed that the protein synthesis ability of the cells was markedly decreased, accompanied by a deficiency in the rough endoplasmic reticulum, leading to progressive loss of pancreatic function, development of pancreatic inflammation and cancer recurrence (11). When ATG7 is specifically knocked out in mouse nerve cells, abnormal protein accumulation and motor function defects appear (12). Autophagy plays an important role in growth and development, and it is the main form of innate immunity in cells, since it plays a role in the defense against the invasion of extracellular microorganisms (13). Furthermore, autophagy is an important mechanism enabling immune cells to survive and serve their roles in tumor microenvironments, which are characterized by poor metabolic conditions and high levels of immunosuppression (14). Autophagy interacts with apoptosis, promoting the mitosis and specific degradation of pro-apoptotic proteins, thereby inhibiting apoptosis. By contrast, after apoptosis is activated, necessary autophagy-related proteins (ARPs) are inactivated or autophagy proteins are converted into apoptotic proteins under certain conditions (15).

Autophagy includes five stages: Induction, nucleation, expansion and containment, fusion and degradation. Mammalian target of rapamycin receptor complex 1 (mTORC1) inhibits autophagy (16). The initiation of cell autophagy requires a variety of $\mathrm{mTORC} 1$ inhibition pathways to be activated to reverse the upstream inhibition of autophagy $(17,18)$. The class III phosphatidylinositol 3-kinase (PI3K) complex constituting of ATG proteins plays an important role in the recruitment and positioning of ATG18 and ATG2, which in turn recruits ATG8, ATG9 and ATG12 to form the pre-autophagosomal structure $(19,20)$. This complex process causes the cargo to gather and nucleate, laying the foundation for the subsequent expansion of the membrane, in which two connected systems participate. The connection between ATG5 and ATG12 requires the participation of the ubiquitin sample connection system (21). This connection product is the complex required for the binding between phosphoethanolamine and microtubule-associated protein 1 light chain 3 (LC3). Finally, the mature autophagosome membrane is fused with the lysosome membrane structure to release the encapsulated cargo into the lysosome (22).

Regulation at the post-translational level and the regulation of transcription factors are involved in the regulation of autophagy. In the absence of amino acids, activation of general control nonderepressible (Gcn)2 (a kinase) and Gcn4 (a transcription factor) promotes ATG gene expression in yeast cells (23). In mammals, the inactivation of mTORC1 promotes the dephosphorylation and translocation of transcription factor $\mathrm{EB}$, and the transcription of various genes associated with the autophagy process (24).

In the absence of energy, autophagy is activated via several pathways, including the AMP-activated protein kinase A pathway, which directly triggers autophagy (25). In addition, it has been found that spermidine inhibits autophagy by inhibiting various acetyl transferases (Fig. 1) (26).

\section{Autophagy and tumors}

Autophagy prevents genes from being altered by reactive oxygen species (ROS) and mitochondria (4), and inhibits the expression of anti-apoptotic proteins to suppress tumorigenesis (27). Autophagy can also restrain tumorigenesis by repressing mitochondrial swelling, p62 accumulation, oxidative stress and genome damage, particularly in mouse liver tumors (5). Nuclear factor-erythroid 2-related factor 2 (NRF2), which can be activated by p62, is mainly responsible for the transcription of anti-oxidation defensive genes, and for facilitating cell survival and tumorigenesis (28). A previous study found that autophagy deficiency by ATG7 deletion promoted p62 accumulation and NRF2 activation. In mouse hepatocarcinoma with autophagy deficiency, knockdown of p62 partially suppressed tumor progression (29), while knockdown of Parkinson disease 2 (a E3 ubiquitin ligase that is responsible for coding the E3 ligase parkin, a multifaceted protein involved in the signaling pathway of mitochondrial autophagy) promoted liver cancer $(28,30)$. Additionally, systemic ATG5 elimination and ATG7 knockdown contribute to the formation of hepatocarcinoma, particularly in the liver of mice (29). In summary, autophagy is associated with the malignant transformation of hepatocytes in mice, and it has been demonstrated to be important for stabilizing cellular homeostasis in tumor suppression.

As a double-edged sword, autophagy has promotive effects on tumorigenesis and tumor growth. It has been found that autophagy is associated with high viability and resistance to antitumor drugs. ROS stimulates autophagy through the Ras (a gene involved in the regulation of various cell signaling pathways)/Raf1 (a serine/threonine kinase)/MEK1/2/ERK1/2 pathway, while it restrains autophagy via activation of the PI3K and AKT (a protein kinase)/mTOR pathway $(31,32)$. Based on the role of autophagy in the regulation of cell genetics and nutrient metabolism, enhanced autophagy may relieve hypoxia and nutrient deficiency during the overgrowth of cancer cells (33). A previous study showed that both ROS and BRAF simultaneously stimulated tumor growth and improved the levels of autophagy (28). In cancer cells with KRAS activation, inhibition of autophagy triggers mitochondrial oxidative phosphorylation and increases ROS production, thus exerting antitumor efficacy $(34,35)$. Deficiency of the autophagy-suppressor gene ATG7 restricts tumor progression and promotes the accumulation of mitochondrial dysfunction through BRAF activation (36). A previous study demonstrated that an autophagy-deficient non-small cell lung cancer (NSCLC)-derived cell line in mice with p53 deficiency was highly dependent on glutamine from the environment for the normal functioning of the mitochondria to support the metabolism and survival in starvation (37). Resistance to vemurafenib, a tumor inhibitor, is linked with active autophagy in patients with melanoma, while the resistance of melanoma to vemurafenib can be reversed by hydroxychloroquine, which was discovered in a drug stimulation trial in a melanoma cell line (38). Furthermore, clinical trials evaluating the potential of autophagy inhibition protocols on brain cancer have indi- 


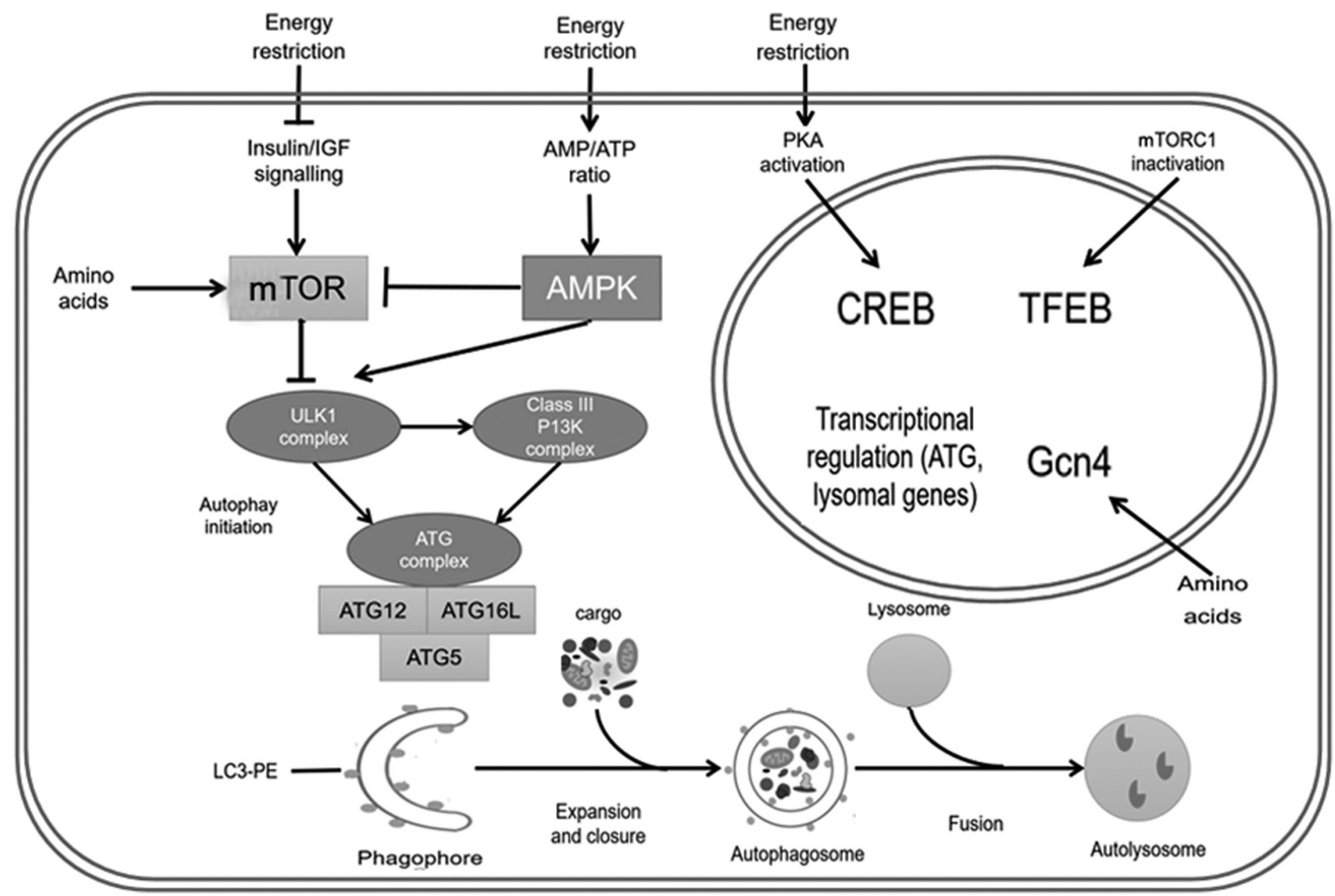

Figure 1. Process and regulation of autophagy. Energy restriction inhibits mTOR and promotes autophagy. Under energy constraints, the increased AMP/ATP ratio activates the AMPK pathway, which either directly promotes autophagy or promotes autophagy by inhibiting the mTOR pathway. Energy restriction also activates CREB protein through the protein kinase A signaling pathway and activates transcription factor EB by inhibiting mTORC1. These autophagy transcription factors will promote the transcription of autophagy-related proteins and lysosomal proteins. Amino acid deficiency activates mTOR and Gcn 4 signaling pathways, and promotes autophagy. mTOR inhibition and AMPK activation promotes the activation of the ULK1 complex upstream of autophagy. The ULK1 complex promotes the formation of the PI3K and ATG complexes, and ultimately promotes the occurrence of autophagy. After the initiation of autophagy, through a series of cascade reactions, the process of expansion and closure occurs, followed by consecutive encircle and fusion steps, which finally leads to the formation of the autolysosome. ULK1, Unc-51-like autophagy activating kinase 1; AMPK, AMP-activated protein kinase; CREB, cAMP-response element binding; PKA, protein kinase A; mTORC1, mammalian target of rapamycin receptor complex 1; Gcn4, general control nonderepressible 4; ATG, autophagy-related gene; TFEB, transcription factor EB; PE, phosphoethanolamine; LC3, microtubule-associated protein 1 light chain 3.

cated that the combined use of chloroquine and vemurafenib prevents the formation of drug resistance (39). Furthermore, autophagy has been found to maintain the intracellular redox balance of tumor stem cells (40), preserve the formation and function of tumor stem cells (41), promote vascular hyperplasia via activation of the receptors of vascular endothelial growth factor (42) and participate in the antigen presentation process of T helper cells (14). These findings indicate that autophagy is beneficial for the survival of tumors, and autophagic inhibition blocks the development of tumors (Fig. 2).

\section{Association between autophagy and RB}

Autophagy and the transformation and occurrence of $R B$. Autophagy is involved in the malignant transformation and gene mutation process of tumors, and the lack of autophagy will lead to the instability of tumor genes, thus promoting the occurrence of tumors (43). RB originates from normal cells, and has been described as a multistep gene variation process of M1, M2or M3 to Mn, with the RB1 allele variation belonging to the M1 to M2 variation process $(44,45)$. It has been clinically found that the RB lesions of certain patients contain a benign tissue region called a retinocytoma $(\mathrm{RC})$. Furthermore, the cell regions of $\mathrm{RC}$ have higher genetic stability than those of RB (46). RC may be an intermediate transition state of $\mathrm{RB}$ or RB degradation, which provides a novel idea for the transformation and occurrence of RB. As a benign lesion of $\mathrm{RB}, \mathrm{RC}$ has a stronger tolerance to chemotherapy, which was initially speculated to be caused by the different roles of autophagy in RC and RB. A study on the role of autophagy in $\mathrm{RC}$ and $\mathrm{RB}$ indicated that the microtubule-associated protein LC3B was present at a high level in the $\mathrm{RC}$ region (RCR) and in retinal cells, while its levels were markedly lower in the adjacent undifferentiated RB region (47). A large number of autophagic vacuoles were found in RCR cells by electron microscopy, but not in the undifferentiated $\mathrm{RB}$ region, which confirmed that autophagy is a more common phenomenon in RCR (47). Similar to RB, RC has the same mutation in the RB1 allele (48). Mutation of the RB1 gene leads to the inhibition of the E2 transcription factor. When the RB1 gene was transduced into human cancer cells deficient in RB protein, the levels of autophagy were increased (49). A previous study reported that silencing RB tumor repression proteins inhibited autophagy induced by etoposide, leading to increased double-stranded 


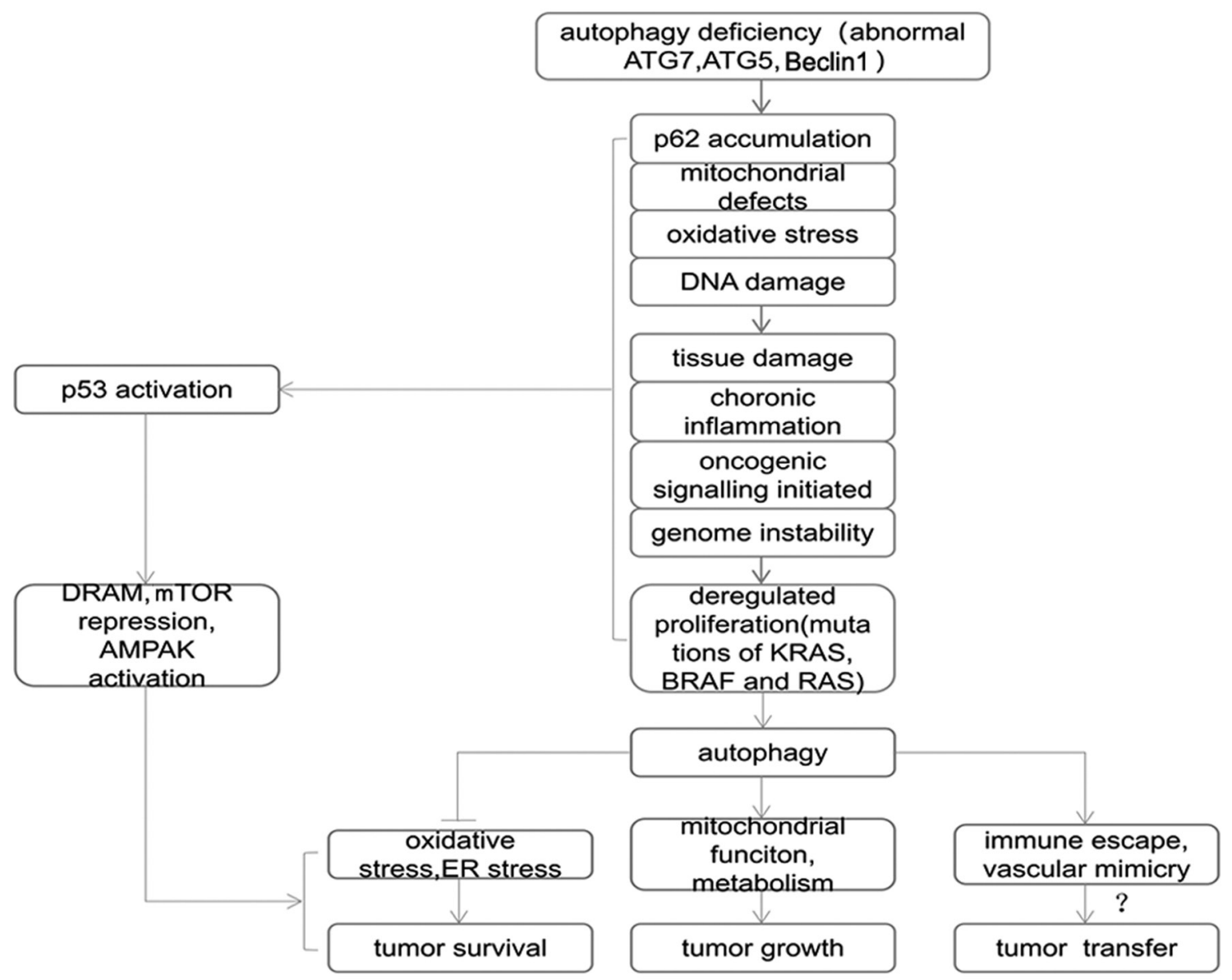

Figure 2. Association between autophagy and tumors. Gene abnormalities lead to autophagy defects in cells by inducing p62 aggregation, mitochondrial defects, oxidative stress, DNA damage and activation of other pathways', which cause tissue damage, chronic inflammation, tumor gene signaling activation and decreased gene stability, which are closely associated with the occurrence of tumors. DNA damage, tumor gene activation and other conditions promote the activation of anti-p53 and other related genes to induce autophagy. Autophagy is used by tumors to promote tumor survival, and may even be a potential mechanism of tumor metastasis. ATG, autophagy-related gene; mTOR, mammalian target of rapamycin; AMPK, AMP-activated protein kinase; ER, endoplasmic reticulum.

DNA damage and tumor cell death (50). RB1 gene mutation reduces the level of autophagy by changing the expression of $\mathrm{RB}$ tumor repression proteins, thus inducing cell gene damage or cell death, which suggests that RC is prone to further M2 to Mn gene mutations, which may be a benign intermediate transition form of RB. Therefore, the decrease in autophagy levels may be one of the critical mechanisms of RB formation. Due to the rarity of RC, the majority of ophthalmologists do not recommend enucleation of the eyeball for patients with $\mathrm{RC}$, and experimental interventional studies on $\mathrm{RC}$ are still relatively scarce. Further studies on the role of RC in the regulation of autophagy may provide important insights into understanding the pathogenesis of RB.

Autophagy and the invasion and metastasis of RB. A previous study suggested a possible link between autophagy and the invasion and metastasis of RB (51). It is recognized that the sesquiterpenoid nootkatone induces the production of endogenous ROS in RB to induce cytotoxicity and inhibit the migration of tumor cells, while it promotes autophagy by promoting the expression of ARPs in RB cells (52). Zhang et al (53) found that p62 and LC3B were highly expressed in the majority of RB tumor cells, and that p53 was expressed in the cytoplasm of certain RB tumor cells. It was also found that high protein levels of p62 and LC3B were significantly associated with latestage TNM and optic nerve invasion in RB, while low levels of $\mathrm{p} 53$ were significantly associated with calcification of RB tumors and optic nerve invasion (53). It is known that p53 is an autophagy inhibitor and LC3B is a marker of autophagy levels; thus, the decreased level of cytoplasmic p 53 and increased level of LC3B can reflect high autophagy levels in RB cells. p62 is degraded by autophagy, and inhibition of autophagy leads to the aggregation of $\mathrm{p} 62$, which is not caused by the abnormal expression of p62; thus, p62 can indirectly reflect the level of autophagy (54). Consequently, it was hypothesized that high levels of autophagy may be one of the mechanisms of RB invasion and metastasis. However, the molecular mechanism of autophagy involved in tumor metastasis and invasion is unclear, and further research is needed.

Autophagy and the resistance and survival of RB. Previous studies have shown that autophagy mediates drug resistance and has a certain protective effect in RB, which indicates the close association between drug resistance and autophagy $(55,56)$. It was found that microRNA (miRNA/miR)-34a regulated apoptosis and autophagy in RB cells by targeting high mobility 


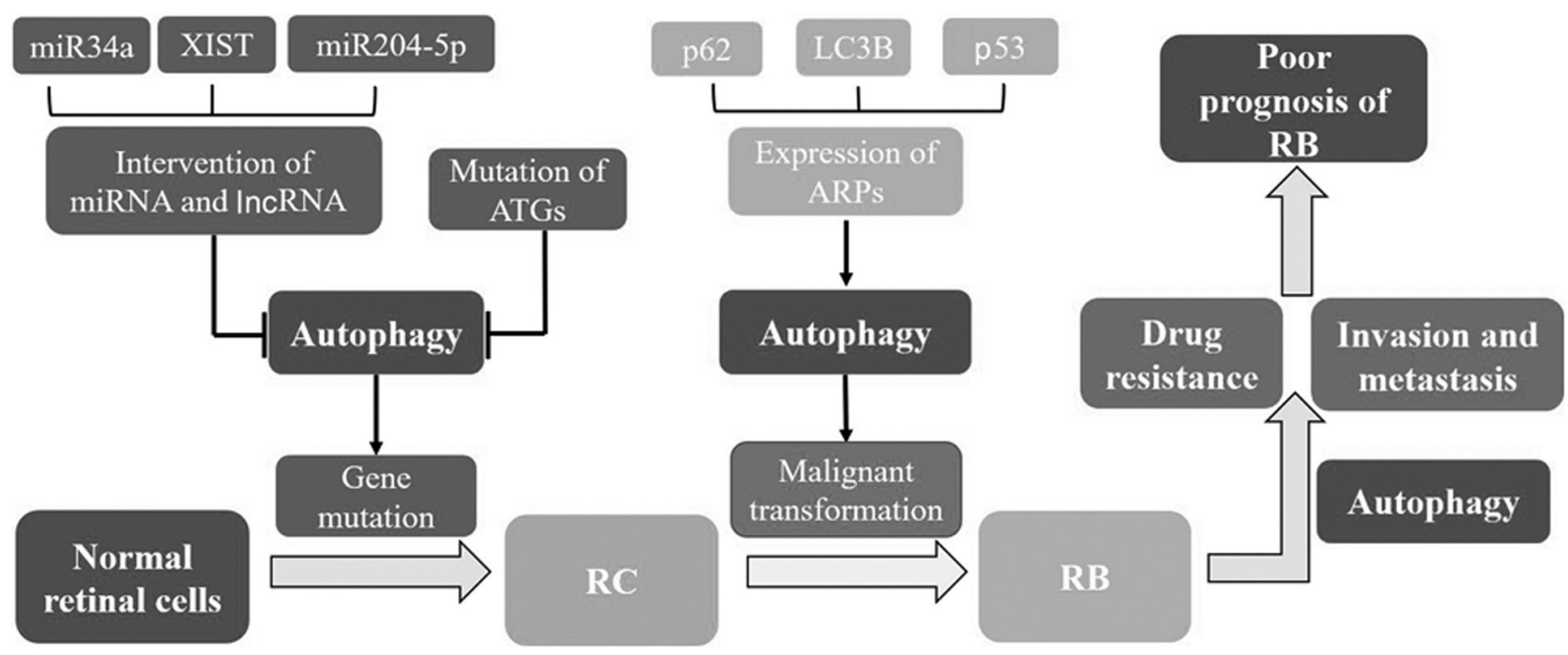

Figure 3. Association between autophagy and RB. Autophagy plays an important role in the occurrence and development of RB via the regulation of various factors such as autophagy-related genes, proteins, various microRNAs and long non-coding RNAs. Inhibition of autophagy promotes gene damage and mutation, thus inducing the formation of retinocytoma (an intermediate transition form). Further genetic variations will lead to malignant transformation and ultimately RB formation. Autophagy is utilized by RB as one of the important mechanisms for drug resistance and invasion, which may be the reason for the poor prognosis of RB. RB, retinoblastoma; RC, retinocytoma; miR, microRNA; XIST, X-inactive specific transcript; lncRNA, long non-coding RNA; ATG, autophagy-related gene; ARP, autophagy-related protein; LC3, microtubule-associated protein 1 light chain 3.

group box 1 (HMGB1), and it was demonstrated that miR-34a inhibited the expression of HMGB1, thus inhibiting autophagy and inducing apoptosis (57). Further results showed that inhibition of autophagy enhanced chemotherapy treatment for DNA damage in RB cells. X-inactive specific transcript (XIST), a 17-kb long non-coding RNA (lncRNA) located on the X chromosome, accelerates tumor progression in certain types of human cancer. Previous studies have demonstrated that the expression of XIST increases in RB tissues and cell lines, and that silencing XIST weakens RB proliferation and autophagy, and enhances the sensitivity to vincristine (VCR) $(58,59)$. Further studies found that XIST sponged miR-204-5p, and that the promoting effect of XIST on RB proliferation and autophagy was reversed by miR-204-5p (59). Thus, it was speculated that decreased miR-204-5p in RB may be associated with drug resistance and proliferation in RB. LINC00152 is increased in RB, and enhances the invasiveness of $\mathrm{RB}$ and its resistance to carboplatin and Adriamycin through the sponging of miR-613; however, silencing LINC00152 inhibits the proliferation, invasiveness and autophagy of RB, and then significantly promotes apoptosis $(60,61)$. The increased level of RB autophagy may be one of the mechanisms of resistance to apoptosis and drug resistance. Huang et al (61) found that metastasis-associated lung adenocarcinoma transcript 1 , as a IncRNA, promoted the autophagy of RB cells by targeting miR-124, thus providing a theoretical basis for drug resistance induced by autophagy. Liu et al (62) applied the autophagy blocker 3-methyladenine (3-MA) to explore the effect of autophagy on RB resistance, and found that the inhibition rate of cisplatin at different concentrations was higher than that observed in tumor cells treated with cisplatin alone, and that the transcription level of drug-resistant genes in cisplatin combined with autophagy inhibitor 3-MA group was markedly downregulated (62). CD24 is a glycosyl phosphatidyl inositol-anchored protein highly expressed in RB tissues and cell lines, and studies have shown that it activates autophagy through the PTEN/AKT/mTORC1 signaling pathway, thereby inactivating the sensitivity of RB to VCR (63). The aforementioned studies suggest that autophagy is a crucial participant in the drug resistance mechanism of $\mathrm{RB}$.

Autophagy is not only associated with chemotherapy resistance in RB, but it may also be an important mechanism for RB survival. Under normal conditions, the expression levels of hypoxia inducible factor-1 (HIF-1) and miR-320 in RB cells are increased compared with those in normal cells. A previous study showed that HIF-1 $\alpha$ was a downstream target of miR-320, and that decreasing miR-320 or HIF-1 $\alpha$ expression inhibited autophagy in RB cells. LC3 aggregation was significantly increased under hypoxia, indicating a high level of autophagy, while inhibition of miR-320 or HIF-1 caused a significant decrease in LC3 aggregation (64). Therefore, it was speculated that increased expression of miR-320 promotes the expression of HIF-1, thereby regulating the expression of ARPs and enhancing RB autophagy. These results suggested that autophagy may be a protective mechanism for $\mathrm{RB}$ to promote its survival in the hypoxic environment of the center ischemic region of the RB. Studies have found that miR-512-3p promotes RB cell apoptosis induced by endoplasmic reticulum stress; however, this process is associated with the inhibition of autophagy (65). Additionally, it was found that inhibiting autophagy and inducing cytotoxicity in RB cells promoted their apoptosis when using a suicide gene therapy with herpes simplex virus type 1 thymidine kinase/ganciclovir (66).

The present review has so far presented a summary of the various protective effects of autophagy in RB at the gene and protein levels. Autophagy can also regulate the intracellular environment of RB, which allocates available materials for decomposition and utilization under conditions of starvation and lack of nutrients, and also helps to maintain the homeostasis of the cytoplasm against stress caused by adverse 
external environments. To date, several studies have reported conflicting results. Ginsenoside Rh2 inhibited RB proliferation but promoted autophagy and apoptosis by downregulating miR-638, while methylbutanol led to potential anti-RB effects by inducing autophagy and cell cycle arrest, and inhibiting the PI3K/mTOR/Akt signaling pathway $(67,68)$. In these studies, the antitumor effect of anticancer drugs or substances may have been accompanied by an increase in the autophagy level, but such studies could not demonstrate whether an elevated level of autophagy is associated with RB repression. On the contrary, the aforementioned drugs with anti-RB effects may induce autophagy and lead to important drug resistance, similar to numerous anti-RB drugs such as etoposide and cisplatin. A number of studies reached the opposite conclusion, and found that dimethyldibutanol 2-methyl-2-butanol (MBT) led to RB cell apoptosis, while the use of autophagy inhibitors blocked the action of this drug (69). These results indicate that the anti-RB effect of MBT may be associated with an increase in the autophagy level, while the specific mechanism remains unknown. Therefore, further studies on the association between autophagy and RB will help us to understand the mechanism of drug resistance in RB (Fig. 3).

\section{Review and summary}

Autophagy, a catabolic process conserved by evolution, plays a vital role in maintaining the homeostasis of the internal and external environments of the cell, and in enhancing the adaptability of the cell to the external environment. The present study reviewed relevant studies on the association between autophagy and RB, and concluded that autophagy plays a 'housekeeping' role in normal, RB and other tumor cells by protecting the cells, promoting the cell fight against external adverse stimuli and improving cell viability. Autophagy degrades damaged intracellular organelles in time and protects DNA stability to maintain normal cell functions in normal cells. Contrary to the situation in normal cells, in tumor cells, autophagy becomes the main mechanism for RB cells to fight against external adverse factors, leading to drug resistance, anti-hypoxia and high tumor invasiveness of these cells. A marked decrease in autophagy in both normal and RB cells has serious consequences. Decreased levels of autophagy lead to apoptosis or promotion of cancer in normal cells, whereas in RB or other tumor cells, they lead to cytotoxic damage and decrease the drug resistance of tumor cells, eventually leading to tumor cell death. Therefore, inhibition of autophagy in RB cells may be an effective target for treatment or may serve as an adjunct therapy to enhance the efficacy of anticancer drugs. The method to specifically target RB tissues or cells without significantly affecting autophagy in normal cells needs to be clarified.

Numerous studies have found that lncRNA and miRNA have significant regulatory effects on autophagy in RB, and various IncRNAs and miRNAs exert significant anti-RB effects by inhibiting autophagy. In addition, the expression of certain IncRNAs and miRNAs in RB showed obvious specificity. These findings suggest that gene therapy targeting lncRNA and miRNA may become a new effective method for RB therapy by regulating autophagy. However, the molecular mechanism of the interaction between autophagy and RB is still unknown, and further research will provide more reliable theories and novel ideas for the clinical treatment of RB.

\section{Acknowledgements}

Not applicable.

\section{Funding}

The present study was supported by grants from the National Natural Science Foundation of China (grant no. 82060250), the Guangxi Science and Technology Plan Project (grant no. AD20238035), the Open Project of Guangxi Key Laboratory of Brain and Cognitive Neuroscience (grant no. GKLBCN20190105-02) and the Guangxi Zhuang Autonomous Region Students' Innovation and Entrepreneurship Training Program of China (grant no. 202010601050).

\section{Availability of data and materials}

Not applicable.

\section{Authors' contributions}

TW and MF wrote the manuscript. ZW, XG and SZ revised it critically for important intellectual content and gave important advice. All authors read and approved the manuscript and agree to be accountable for all aspects of the research in ensuring that the accuracy or integrity of any part of the work are appropriately investigated and resolved. Data authentication is not applicable.

\section{Ethics approval and consent to participate}

Not applicable.

\section{Patient consent for publication}

Not applicable.

\section{Competing interests}

The authors declare that they have no competing interests.

\section{References}

1. Fabian ID, Onadim Z, Karaa E, Duncan C, Chowdhury T, Scheimberg I, Ohnuma SI, Reddy MA and Sagoo MS: The management of retinoblastoma. Oncogene 37: 1551-1560, 2018.

2. Mallipatna A, Marino $M$ and Singh AD: Genetics of Retinoblastoma. Asia Pac J Ophthalmol (Phila) 5: 260-264, 2016.

3. Doherty J and Baehrecke EH: Life, death and autophagy. Nat Cell Biol 20: 1110-1117, 2018.

4. Bartsch K, Knittler K, Borowski C, Rudnik S, Damme M, Aden K, Spehlmann ME, Frey N, Saftig P, Chalaris A, et al: Absence of RNase $\mathrm{H} 2$ triggers generation of immunogenic micronuclei removed by autophagy. Hum Mol Genet 26: 3960 3972, 2017.

5. Takamura A, Komatsu M, Hara T, Sakamoto A, Kishi C, Waguri S, Eishi Y, Hino O, Tanaka K and Mizushima N: Autophagy-deficient mice develop multiple liver tumors. Genes Dev 25: 795-800, 2011.

6. Kimmelman AC and White E: Autophagy and tumor metabolism. Cell Metab 25: 1037-1043, 2017. 
7. Poillet-Perez L and White E: Role of tumor and host autophagy in cancer metabolism. Genes Dev 33: 610-619, 2019.

8. Eskelinen EL: Autophagy: Supporting cellular and organismal homeostasis by self-eating. Int J Biochem Cell Biol 111: 1-10, 2019.

9. Zhou S, Chen X, Xue R, Zhou Q, Hu P, Ouyang X, Dai T, Zhu W and Tian S: Autophagy is involved in the pathogenesis of experimental autoimmune neuritis in rats. Neuroreport 27: 337-344, 2016.

10. Parzych KR and Klionsky DJ: An overview of autophagy: Morphology, mechanism, and regulation. Antioxid Redox Signal 20: 460-473, 2014

11. Antonucci L, Fagman JB, Kim JY, Todoric J, Gukovsky I, Mackey M, Ellisman MH and Karin M: Basal autophagy maintains pancreatic acinar cell homeostasis and protein synthesis and prevents ER stress. Proc Natl Acad Sci USA 112: E6166-E6174, 2015

12. Honda S, Arakawa S, Yamaguchi H, Torii S, Tajima Sakurai H, Tsujioka M, Murohashi M and Shimizu S: Association between Atg5-independent alternative autophagy and neurodegenerative diseases. J Mol Biol 432: 2622-2632, 2020.

13. Keller MD, Torres VJ and Cadwell K: Autophagy and microbial pathogenesis. Cell Death Differ 27: 872-886, 2020.

14. Rybstein MD, Bravo-San Pedro JM, Kroemer G and Galluzzi L: The autophagic network and cancer. Nat Cell Biol 20: 243-251, 2018.

15. Mariño G, Niso-Santano M, Baehrecke EH and Kroemer G: Self-consumption: The interplay of autophagy and apoptosis. Nat Rev Mol Cell Biol 15: 81-94, 2014.

16. Laplante M and Sabatini DM: mTOR signaling in growth control and disease. Cell 149: 274-293, 2012.

17. Nazio F, Strappazzon F, Antonioli M, Bielli P, Cianfanelli V, Bordi M, Gretzmeier C, Dengjel J, Piacentini M, Fimia GM, et al: mTOR inhibits autophagy by controlling ULK1 ubiquitylation, self-association and function through AMBRA1 and TRAF6. Nat Cell Biol 15: 406-416, 2013

18. Li Z, Tian X, Ji X, Wang J, Chen H, Wang D and Zhang X ULK1-ATG13 and their mitotic phospho-regulation by CDK1 connect autophagy to cell cycle. PLoS Biol 18: e3000288, 2020.

19. Araki Y, Ku WC, Akioka M, May AI, Hayashi Y, Arisaka F, Ishihama Y and Ohsumi Y: Atg38 is required for autophagyspecific phosphatidylinositol 3-kinase complex integrity. J Cell Biol 203: 299-313, 2013.

20. Kotani T, Kirisako H, Koizumi M, Ohsumi Y and Nakatogawa $H$ : The Atg2-Atg18 complex tethers pre-autophagosomal membranes to the endoplasmic reticulum for autophagosome formation. Proc Natl Acad Sci USA 115: 10363-10368, 2018.

21. Hanada T, Noda NN, Satomi Y, Ichimura Y, Fujioka Y, Takao T, Inagaki $\mathrm{F}$ and Ohsumi Y: The Atg12-Atg5 conjugate has a nove E3-like activity for protein lipidation in autophagy. J Biol Chem 282: 37298-37302, 2007.

22. Guo H, Chitiprolu M, Roncevic L, Javalet C, Hemming FJ, Trung MT, Meng L, Latreille E, Tanese de Souza C, McCulloch D, et al: Atg5 Disassociates the V1V0-ATPase to Promote exosome production and tumor metastasis independent of canonical macroautophagy. Dev Cell 43: 716-730.e7, 2017.

23. B'Chir W, Maurin AC, Carraro V, Averous J, Jousse C, Muranishi Y, Parry L, Stepien G, Fafournoux P and Bruhat A: The eIF2 $\alpha /$ ATF4 pathway is essential for stress-induced autophagy gene expression. Nucleic Acids Res 41: 7683-7699, 2013.

24. Settembre C, Di Malta C, Polito VA, Garcia Arencibia M, Vetrini F, Erdin S, Erdin SU, Huynh T, Medina D, Colella P, et al: TFEB links autophagy to lysosomal biogenesis. Science 332: 1429-1433, 2011.

25. Russell RC, Yuan HX and Guan KL: Autophagy regulation by nutrient signaling. Cell Res 24: 42-57, 2014

26. Pietrocola F, Lachkar S, Enot DP, Niso-Santano M, Bravo-San Pedro JM, Sica V, Izzo V, Maiuri MC, Madeo F, Mariño G, et al: Spermidine induces autophagy by inhibiting the acetyltransferase EP300. Cell Death Differ 22: 509-516, 2015.

27. Wang Y, Zhang N, Zhang L, Li R, Fu W, Ma K, Li X, Wang L, Wang J, Zhang H, et al: Autophagy regulates chromatin ubiquitination in DNA damage response through elimination of SQSTM1/p62. Mol Cell 63: 34-48, 2016.

28. Huang T, Song X, Yang Y, Wan X, Alvarez AA, Sastry N, Feng H, $\mathrm{Hu}$ B and Cheng SY: Autophagy and Hallmarks of Cancer. Crit Rev Oncog 23: 247-267, 2018.

29. Takamura A, Komatsu M, Hara T, Sakamoto A, Kishi C, Waguri S, Eishi Y, Hino O, Tanaka K and Mizushima N: Autophagy-deficient mice develop multiple liver tumors. Genes Dev 25: 795-800, 2011
30. Poulogiannis G, McIntyre RE, Dimitriadi M, Apps JR, Wilson $\mathrm{CH}$, Ichimura K, Luo F, Cantley LC, Wyllie AH, Adams DJ, et al: PARK2 deletions occur frequently in sporadic colorectal cancer and accelerate adenoma development in Apc mutant mice. Proc Natl Acad Sci USA 107: 15145-15150, 2010.

31. Li L, Ishdorj G and Gibson SB: Reactive oxygen species regulation of autophagy in cancer: Implications for cancer treatment Free Radic Biol Med 53: 1399-1410, 2012.

32. Pattingre S, Bauvy $\mathrm{C}$ and Codogno P: Amino acids interfere with the ERK1/2-dependent control of macroautophagy by controlling the activation of Raf-1 in human colon cancer HT-29 cells. J Biol Chem 278: 16667-16674, 2003.

33. White E: The role for autophagy in cancer. J Clin Invest 125: 42-46, 2015.

34. Yang S, Wang X, Contino G, Liesa M, Sahin E, Ying H, Bause A, Li Y, Stommel JM, Dell'antonio G, et al: Pancreatic cancers require autophagy for tumor growth. Genes Dev 25: 717-729, 2011.

35. Guo JY, Chen HY, Mathew R, Fan J, Strohecker AM, KarsliUzunbas G, Kamphorst JJ, Chen G, Lemons JM, Karantza V, et al: Activated Ras requires autophagy to maintain oxidative metabolism and tumorigenesis. Genes Dev 25: 460-470, 2011.

36. Strohecker AM and White E: Autophagy promotes BrafV600Edriven lung tumorigenesis by preserving mitochondrial metabolism. Autophagy 10: 384-385, 2014.

37. Guo JY, Karsli-Uzunbas G, Mathew R, Aisner SC, Kamphorst JJ, Strohecker AM, Chen G, Price S, Lu W, Teng X, et al: Autophagy suppresses progression of K-ras-induced lung tumors to oncocytomas and maintains lipid homeostasis. Genes Dev 27: $1447-1461,2013$

38. Ma XH, Piao SF, Dey S, McAfee Q, Karakousis G, Villanueva J, Hart LS, Levi S, Hu J, Zhang G, et al: Targeting ER stress-induced autophagy overcomes BRAF inhibitor resistance in melanoma. J Clin Invest 124: 1406-1417, 2014.

39. Levy JM, Thompson JC, Griesinger AM, Amani V, Donson AM, Birks DK, Morgan MJ, Mirsky DM, Handler MH, Foreman NK, et al: Autophagy inhibition improves chemosensitivity in BRAF(V600E) brain tumors. Cancer Discov 4: 773-780, 2014.

40. Perera RM, Stoykova S, Nicolay BN, Ross KN, Fitamant J, Boukhali M, Lengrand J, Deshpande V, Selig MK, Ferrone CR, et al: Transcriptional control of autophagy-lysosome function drives pancreatic cancer metabolism. Nature 524: 361-365, 2015.

41. Liu K, Lee J, Kim JY, Wang L, Tian Y, Chan ST, Cho C, Machida K, Chen D and Ou JJ: Mitophagy controls the activities of tumor suppressor p53 to regulate hepatic cancer stem cells. Mol Cell 68: 281-292.e5, 2017.

42. Wu HB, Yang S, Weng HY, Chen Q, Zhao XL, Fu WJ, Niu Q, Ping YF, Wang JM, Zhang X, et al: Autophagy-induced KDR/ VEGFR-2 activation promotes the formation of vasculogenic mimicry by glioma stem cells. Autophagy 13: 1528-1542, 2017.

43. Amaravadi RK, Kimmelman AC and Debnath J: Targeting autophagy in cancer: Recent advances and future directions. Cancer Discov 9: 1167-1181, 2019.

44. Soliman SE, Racher H, Zhang C, MacDonald H and Gallie BL: Genetics and Molecular Diagnostics in Retinoblastoma - An Update. Asia Pac J Ophthalmol (Phila) 6: 197-207, 2017.

45. Laurie NA, Donovan SL, Shih CS, Zhang J, Mills N, Fuller C, Teunisse A, Lam S, Ramos Y, Mohan A, et al: Inactivation of the p53 pathway in retinoblastoma. Nature 444: 61-66, 2006.

46. Dimaras H, Khetan V, Halliday W, Orlic M, Prigoda NL, Piovesan B, Marrano P, Corson TW, Eagle RC Jr, Squire JA, et al: Loss of RB1 induces non-proliferative retinoma: Increasing genomic instability correlates with progression to retinoblastoma. Hum Mol Genet 17: 1363-1372, 2008.

47. Liu Y, Wan ST, Zhang P, Zhang WX, Zheng JL, Lin JX and Li YP: Expression levels of autophagy related proteins and their prognostic significance in retinocytoma and retinoblastoma. Int $\mathrm{J}$ Ophthalmol 7: 594-601, 2014.

48. Indovina P, Acquaviva A, De Falco G, Rizzo V, Onnis A, Luzzi A, Giorgi F, Hadjistilianou T, Toti P, Tomei V, et al: Downregulation and aberrant promoter methylation of p16INK4A: A possible novel heritable susceptibility marker to retinoblastoma. J Cell Physiol 223: 143-150, 2010

49. Jiang H, Martin V, Gomez-Manzano C, Johnson DG, Alonso M, White E, Xu J, McDonnell TJ, Shinojima N and Fueyo J: The RB-E2F1 pathway regulates autophagy. Cancer Res 70: 7882-7893, 2010. 
50. Biasoli D, Kahn SA, Cornélio TA, Furtado M, Campanati L, Chneiweiss H, Moura-Neto V and Borges HL: Retinoblastoma protein regulates the crosstalk between autophagy and apoptosis, and favors glioblastoma resistance to etoposide. Cell Death Dis 4: e767, 2013.

51. Chen $\mathrm{N}$ and Karantza-Wadsworth V: Role and regulation of autophagy in cancer. Biochim Biophys Acta 1793: 1516-1523, 2009.

52. Zhu X, Li X and Chen Z: Inhibition of anticancer growth in Retinoblastoma cells by naturally occurring sesquiterpene nootkatone is mediated via autophagy, endogenous ROS production, cell cycle arrest and inhibition of NF- $\mathrm{KB}$ signalling pathway. J BUON 25: 427-431, 2020.

53. Zhang M, Zhou YF, Gong JY, Gao CB and Li SL: Expression of autophagy-related protein LC3B, p62, and cytoplasmic p53 in human retinoblastoma tissues. Eur Rev Med Pharmacol Sci 20 3152-3160, 2016.

54. Komatsu $\mathrm{M}$ and Ichimura $\mathrm{Y}$ : Physiological significance of selective degradation of p62 by autophagy. FEBS Lett 584 1374-1378, 2010.

55. Li YJ, Lei YH, Yao N, Wang CR, Hu N, Ye WC, Zhang DM and Chen ZS: Autophagy and multidrug resistance in cancer. Chin J Cancer 36: 52, 2017

56. Yoshida GJ: Therapeutic strategies of drug repositioning targeting autophagy to induce cancer cell death: From pathophysiology to treatment. J Hematol Oncol 10: 67, 2017.

57. Cai CL, Jin L, Lang XL and Li BL: Long noncoding RNA XIST regulates cardiomyocyte apoptosis by targeting miR-873-5p/ MCL1 axis. Eur Rev Med Pharmacol Sci 24: 12878-12886, 2020.

58. Liu K, Huang J, Xie M, Yu Y, Zhu S, Kang R, Cao L, Tang D and Duan X: MIR34A regulates autophagy and apoptosis by targeting HMGB1 in the retinoblastoma cell. Autophagy 10: 442-452, 2014

59. Yao L, Yang L, Song H, Liu TG and Yan H: Silencing of lncRNA XIST suppresses proliferation and autophagy and enhances vincristine sensitivity in retinoblastoma cells by sponging miR204-5p. Eur Rev Med Pharmacol Sci 24: 3526-3537, 2020.
60. Wang Y, Xin D, Zhou L: LncRNA LINC00152 increases the aggressiveness of human retinoblastoma and enhances carboplatin and adriamycin resistance by regulating miR-613/ Yes-associated protein 1 (YAP1) axis. Med Sci Monit 26: e920886-1-e920886-17, 2020.

61. Huang J, Yang Y, Fang F and Liu K: MALAT1 modulates the autophagy of retinoblastoma cell through miR-124-mediated stx17 regulation. J Cell Biochem 119: 3853-3863, 2018.

62. Liu Y, Su J and Wang LH: Role of autophagy in cisplatin resistance of retinoblastoma Y79 cells and its mechanism. Zhongliu Fangzhi Yanjiu 45: 517-522, 2018.

63. Sun J, Feng D, Xi H, Luo J, Zhou Z, Liu Q, Chen Y and Shao Q: CD24 blunts the sensitivity of retinoblastoma to vincristine by modulating autophagy. Mol Oncol 14: 1740-1759, 2020.

64. Liang $Y$, Chen $X$ and Liang Z: MicroRNA-320 regulates autophagy in retinoblastoma by targeting hypoxia inducible factor-1 $\alpha$. Exp Ther Med 14: 2367-2372, 2017.

65. Kong M, Han Y, Zhao Y, Zhang H: miR-512-3p overcomes resistance to cisplatin in retinoblastoma by promoting apoptosis induced by endoplasmic reticulum stress. Med Sci Monit 26 e923817-1-e923817-10, 2020

66. Yi QY, Bai ZS, Cai B, Chen N, Chen LS, Yuan T and Mao JH: HSV-TK/GCV can induce cytotoxicity of retinoblastoma cells through autophagy inhibition by activating MAPK/ERK. Oncol Rep 40: 682-692, 2018.

67. Li M, Zhang D, Cheng J, Liang J and Yu F: Ginsenoside Rh2 inhibits proliferation but promotes apoptosis and autophagy by down-regulating microRNA-638 in human retinoblastoma cells. Exp Mol Pathol 108: 17-23, 2019.

68. Yin L, Sun Z, Ren Q, Su X and Zhang D: Methyl eugenol induces potent anticancer effects in RB355 human retinoblastoma cells by inducing autophagy, cell cycle arrest and inhibition of PI3K/ mTOR/Akt signalling pathway. J BUON 23: 1174-1178, 2018

69. Li X, Zhu X, Xu C and Wu J: 2-Methyl 2-butanol suppresses human retinoblastoma cells through cell cycle arrest and autophagy. Braz J Med Biol Res 51: e6889, 2018. 